Gastroenterologe 2018 $13: 262-263$ https://doi.org/10.1007/s11377-018-0269-2 Online publiziert: 17. Mai 2018 (c) Springer Medizin Verlag GmbH, ein Teil von Springer Nature 2018

CrossMark

\section{Friedrich-Rust ${ }^{1} \cdot$ M. Gebel $^{2}$}

'Medizinische Klinik 1, Universitätsklinikum Frankfurt, Frankfurt, Deutschland

${ }^{2}$ Klinik für Gastroenterologie, Hepatologie und Endokrinologie, Medizinische Hochschule Hannover, Hannover, Deutschland

\title{
Ultraschalldiagnostik im Jahr 2018
}

\section{Stellenwert und Herausforderungen}

Die ultraschallbasierte Elastographie der Leber ist inzwischen ein fest etabliertes Verfahren, das bei Patienten mit chronischer Lebererkrankung zur Beurteilung des Leberfibrosestadiums und zur Prognoseeinschätzung ergänzend zur konventionellen Sonographie angewandt wird. Inzwischen bieten fast alle Ultraschallfirmen eine Elastographie integriert in Routineultraschallgeräte an. Viola Knop erläutert in ihrem Beitrag die Unterschiede der Systeme und gibt Tipps zur Interpretation in der klinischen Praxis. Die nichtalkoholische Fettlebererkrankung (NAFLD) als Ursache einer chronischen Lebererkrankung mit ihren Folgeerkrankungen nimmt auch in Deutschland zu. Zur Diagnosesicherung gilt immer noch die Leberbiopsie als Goldstandard, jedoch ist die Akzeptanz unter den Patienten gering. Zudem ist auch die Leberhistologie für die Beurteilung des Leberfibrosestadiums nicht perfekt und wahrscheinlich den nichtinvasiven Verfahren nicht überlegen. Dies $\mathrm{zu}$ beweisen oder $\mathrm{zu}$ widerlegen, ist bei fehlendem besserem Goldstandard schwer. Die Steastosebeurteilung ist inzwischen auch durch den „controlled attenuation parameter", der die sonographische Schallabschwächung quantifiziert, nichtinvasiv durchführbar. Für die Beurteilung der Inflammation ist die Leberhistologie jedoch derzeit noch besser als Laborwerte und nichtinvasive Verfahren. Patienten mit NAFLD haben auch ohne das Vorliegen einer Leberzirrhose ein erhöhtes Risiko, ein hepatozelluläres Karzinom (HCC) zu entwickeln, sodass halbjährlich eine So- nographie zum Screening empfohlen wird. Dies stellt eine logistische und qualitative Herausforderung dar. Auch hier können nichtinvasive Verfahren in der Selektion von Risikopatienten helfen. Thomas Karlas diskutiert in seinem Beitrag die Bedeutung der sonographischen Verfahren für die Diagnose und Verlaufsbeurteilung von NAFLD-Patienten. Liegt bereits ein Lebertumor vor, sind neben der Operation und Chemotherapie die perkutanen lokal ablativen Therapieverfahren primärer oder sekundärer maligner Lebertumoren etablierte Verfahren in der Onkologie. Philipp Kasten und Andrej Potthoff berichten in ihrem Beitrag über die Techniken und diskutieren die Vorteile der sonographisch assistierten Intervention.

Voraussetzung für eine erfolgreiche Therapie ist die rechtzeitige Diagnose eines malignen Lebertumors. In der deutschlandweiten Multizenterstudie der Deutschen Gesellschaft für Ultraschall in der Medizin (DEGUM) zur Charakterisierung von unklaren Lebertumoren mit kontrastverstärktem Ultraschall (CEUS) hatte sich bei Subgruppenanalysen gezeigt, dass sich die CEUS bei der Diagnose von malignen Lebertumoren hinsichtlich der Sensitivität und Spezifität nicht signifikant von der Magnetresonanztomographie (MRT) und Computertomographie (CT) unterschied. Alle Verfahren haben aber auch Schwächen bei untypischem Kontrastverhalten. Rüdiger Görtz und Barbara Schellhaas vergleichen den für statische Verfahren, wie CT und MRT, entwickelten Diagnosealgorithmus des American College of Radiology mit dem Erlanger Diagnosealgorithmus. Diese Diagnosealgorithmen sollen die Sicherheit der bildgebenden Diagnose transparent machen. Bei allen auch mit diesen Algorithmen unklar bleibenden Tumoren kann die Diagnose nur mit dem Goldstandard der Tumormorphologie gestellt werden. Die transkutane oder endosonographische ultraschallgeleitete Biopsie erlaubt eine elegante minimalinvasive Probengewinnung. Leider wird dabei organabhängig in 10-30 \% der Fälle kein repräsentatives oder diagnostisch ausreichendes Material gewonnen. Michael Hocke zeigt die Möglichkeiten der Optimierung der Materialgewinnung bis hin zur On-site-Zytologie auf.

Die Sonographie gilt als untersucherabhängig. Dies ist insofern richtig, weil die Bilderhebung nicht maschinell, sondern durch den Untersucher erfolgt. Ein dafür nötiges Untersuchungsgeschick und räumliches Vorstellungsvermögen (American College of Emergency Physicians [ACEP]: „psychomotoric skills“) müssen trainiert werden. $\mathrm{Zu}$ den neuen Möglichkeiten, diese „skills“ auch bei typischen oder ungewöhnlichen sonographischen Befunden zu trainieren und zu entwickeln, gehören Simulatoren mit originalen räumlichen Patientendaten. Unter der Bedingung, dass Untersucher in Methodenvergleichsstudien auch gleiche Qualität haben, wurde schon früher in einer brillanten Studie [1] gezeigt, dass die Sonographie bei der Detektion primärer Lebertumoren bei Patienten auf der Lebertransplantionsliste im Vergleich zur CT und MRT und dem Explantat bei hoher Diagnosesicherheit die gerings- 
te Untersucherabhängigkeit aufwies. Darmerkrankungen stellen noch höhere Ansprüche, da das Organ teils gasgefüllt und mobil ist. Radiologische Verfahren wie die CT sind durch die Strahlenbelastung bei komplizierten Verläufen kompromittiert und werden in Leitlinien nicht mehr empfohlen. Die in Leitlinien noch empfohlene MRT ist wegen des Risikos der Gadoliniumablagerung im Gehirn auch bei zyklischen Präparationen bei Wiederholungsuntersuchungen nicht mehr als unbedenklich anzusehen. Damit werden auch Zulassungsstudien neuer Medikamente für die Therapie entzündlicher Darmerkrankungen mit diesen Methoden problematisch. Die hochauflösende Sonographie des Darms hat sich schon für die Diagnose der Appendizitis und Divertikulitis weltweit bewährt. Im Artikel von Michael Gebel werden robuste sonographische Kriterien der Darmbeteiligung bei M. Crohn, ihre prognostische Bedeutung und ihr Wert für das Therapiemonitoring durch den niedergelassenen Gastroenterologen vorgestellt.

\section{Friednch-Rust}

Prof. Dr. M. Friedrich Rust

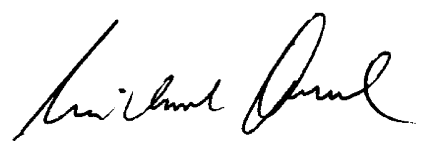

Prof. Dr. M. Gebel

\section{Korrespondenzadresse}

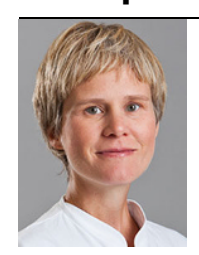

Prof. Dr. M. Friedrich-Rust

Medizinische Klinik 1, Universitätsklinikum Frankfurt

Theodor-Stern-Kai 7, 60590 Frankfurt, Deutschland mireen.friedrich-Rust@kgu.de

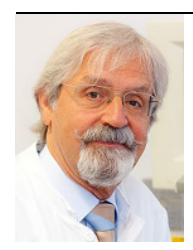

Prof. Dr. M. Gebel

Klinik für Gastroenterologie, Hepatologie und Endokrinologie, Medizinische Hochschule Hannover

Carl-Neuberg-Str. 1, 30625 Hannover, Deutschland gebel.michael@ mh-hannover.de

Interessenkonflikt. M. Friedrich-Rust und M. Gebel geben an, dass kein Interessenkonflikt besteht.

\section{Literatur}

1. Teefey SA (2003) Detection of primary hepatic malignancy in liver transplant candidates: prospective comparison of $\mathrm{CT}$, MR imaging, US, and PET.Radiology 226(2):533-542

\section{Neues Lernportal für Kontrast- mittelsonographie}

Die Kontrastmittelsonographie ist mittlerweile an immer mehr Kliniken im deutschsprachigen Raum verfügbar. Allerdings sind die meisten Angebote zum Erlernen dieser Technik kostenpflichtig. Die Klinik für Gastroenterologie, Hepatologie und gastroenterologische Onkologie des Städtischen Klinikum MünchenBogenhausen stellt jetzt ein neues OnlineLernportal zur Verfügung. Auf der Seite www.kontrastmittelsonographie.de können alle Interessierten Hintergrundwissen zur Methode, einen Videoatlas pathologischer Befunde und regelmäßig aktualisierte Kasuistiken mit internistischgastroenterologischem Schwerpunkt finden - und das kostenfrei und ohne erforderliche Registrierung.

Die Seite ist so aufgebaut, dass man im Bereich Grundlagen die Anwendungsgebiete und deren Besonderheiten kennenlernen kann. Die Lerntexte sind kurz gehalten und wurden nach aktueller Studienlage und unter Berücksichtigung verfügbarer Leitlinien erstellt. Anschließend lässt sich im Videoatlas der typische kontrastmittel-sonographische Befund eines Krankheitsbildes nachvollziehen. Im Bereich Kasuistiken hat der Nutzer die Möglichkeit, sein Wissen im Rahmen aufbereiteter Fallberichte zu überprüfen. Die Sektionen umfassen bereits jetzt mehr als 60 Videos in Originalqualität ergänzt durch hochauflösendes Bildmaterial aus B-Bildund Dopplersonographie sowie passende Schnittbildgebung. Alle verwendeten kontrastmittelsonographischen Diagnosen wurden durch Referenzbildgebung und/oder histopathologisch bestätigt, was ein hohes Maß an Qualität sicherstellt.

Quelle: Klinik für Gastroenterologie, Hepatologie und gastroenterologische Onkologie, Städtisches Klinikum MünchenBogenhausen 